\title{
The relationship between patterns of intermittent hypoxia and retinopathy of prematurity in preterm infants
}

\author{
Juliann M. Di Fiore' ${ }^{1}$, Farhad Kaffashi ${ }^{2}$, Kenneth Loparo², Abdus Sattar ${ }^{3}$, Mark Schluchter ${ }^{3}$, Ryan Foglyano' ${ }^{1}$, Richard J. Martin ${ }^{1}$ \\ and Christopher G. Wilson'
}

BACKGROUND: We have previously shown an increased incidence of intermittent hypoxemia $(\mathrm{IH})$ events in preterm infants with severe retinopathy of prematurity (ROP). Animal models suggest that patterns of IH events may play a role in ROP severity as well. We hypothesize that specific $\mathrm{IH}$ event patterns are associated with ROP in preterm infants.

METHODS: Variability in $\mathrm{H}$ event duration, severity, and the time interval between $\mathbb{H}$ events $(\leq 80 \%, \geq 10 \mathrm{~s}$, and $\leq 3 \mathrm{~min}$ ) along with the frequency spectrum of the oxygen saturation $\left(\mathrm{SpO}_{2}\right)$ waveform were assessed.

RESULTS: Severe ROP was associated with (i) an increased mean and SD of the duration of $\mathrm{H}$ event $(P<0.005)$, (ii) more variability (histogram entropy) of the time interval between $\mathrm{IH}$ events $(P<0.005)$, (iii) a higher IH nadir $(P<0.05)$, (iv) a time interval between $1 \mathrm{H}$ events of $1-20 \mathrm{~min}(P<0.05)$, and $(v)$ increased spectral power in the range of $0.002-0.008 \mathrm{~Hz}(P<0.05)$, corresponding to $\mathrm{SpO}_{2}$ waveform oscillations of $2-8 \mathrm{~min}$ in duration. Spectral differences were detected as early as $14 \mathrm{~d}$ of life.

CONCLUSION: Severe ROP was associated with more variable, longer, and less severe IH events. Identification of specific spectral components in the $\mathrm{SpO}_{2}$ waveform may assist in early identification of infants at risk for severe ROP.

$\mathbf{R}^{\mathrm{s}}$ etinopathy of prematurity (ROP), a vasoproliferative disorder of the retina that can produce significant loss of vision, continues to cause major morbidity in the preterm population. Reduction in oxygen levels has been only partially successful in reducing the incidence of this disease (1-3). The first phase of ROP is associated with hyperoxia, which is thought to downregulate vascular endothelial growth factor and initiate avascularization or vascular regression. In the second phase, the retina becomes hypoxic, upregulating vascular endothelial growth factor and other growth factors, leading to rebound neovascularization. In animal models, short fluctuations in oxygen levels also play a role in neovascularization and retinal levels of vascular endothelial growth factor (4-6).

We have previously shown that intermittent hypoxemia (IH) events are associated with severe ROP in preterm infants. In neonates of 24-28wk gestation, the occurrence of IH events increases with advancing postnatal age. In infants with mild or no ROP, the incidence of IH events is relatively low during the first week of life, increases through the fifth week of life, followed by a decrease in IH events thereafter (7). In contrast, after $5 \mathrm{wk}$ of age, the prevalence of IH events remains elevated in infants with severe ROP. Anecdotally, there is a wide range in the patterns of these events both within and between infants, as IH events can occur in isolated episodes or clusters such as those accompanying periodic breathing. Timing of IH events may affect the extent of neovascularization. Rodent data have shown that clustered IH events result in increased neovascularization and higher levels of retinal vascular endothelial growth factor as compared with a comparable number of equally dispersed IH events (4). Therefore, the purpose of this study was to characterize the patterns of $\mathrm{IH}$ events associated with severe $\mathrm{ROP}$ in preterm infants.

\section{RESULTS}

The study population included 63 infants with mild or no ROP and 16 infants with severe ROP requiring laser therapy. As previously reported (7), the infants with severe ROP had lower birth weights ( $730 \pm 156$ vs. $863 \pm 180 \mathrm{~g}, P<0.01)$, lower gestational age ( $25.5 \pm 0.7$ vs. $26.2 \pm 1.2 \mathrm{wk}, P<0.001)$, and had a higher percentage of males ( 69 vs. $36 \%, P<0.05$ ). There was no difference between infant groups in multiple births ( $25 \mathrm{vs}$. $21 \%$ ) or race (63 vs. $41 \%$ Caucasian). The infants with severe ROP received more days of supplemental oxygen therapy (51 \pm 12 vs. $34 \pm 20 \mathrm{~d}, P<0.001)$ and ventilatory support $(40 \pm 18$ vs. $14 \pm 15 \mathrm{~d}, P<0.001)$ and had fewer days on caffeine (34 \pm 16 vs. $44 \pm 12 \mathrm{~d}, P<0.01)$; there was a higher incidence of $\mathrm{IH}$ events in the severe ROP group after $35 \mathrm{~d}$ of life (7).

Given that the higher number of IH episodes might be attributed to lower mean oxygen saturation in the ROP group, both the mean oxygen saturation and the mean baseline oxygen saturation, excluding $\mathrm{IH}$ events, were calculated. Mean and baseline oxygen saturation levels were significantly lower in the ROP group $(P<0.05$ and $P<$ 0.01 , respectively, Figure 1$)$ after $42 \mathrm{~d}$ of life $(P<0.05)$. Of note, infants with severe ROP had decreased event severity $(P=0.04)$, as defined by a higher nadir, after $14 \mathrm{~d}$ of life $(P<0.05$, Figure 2). 

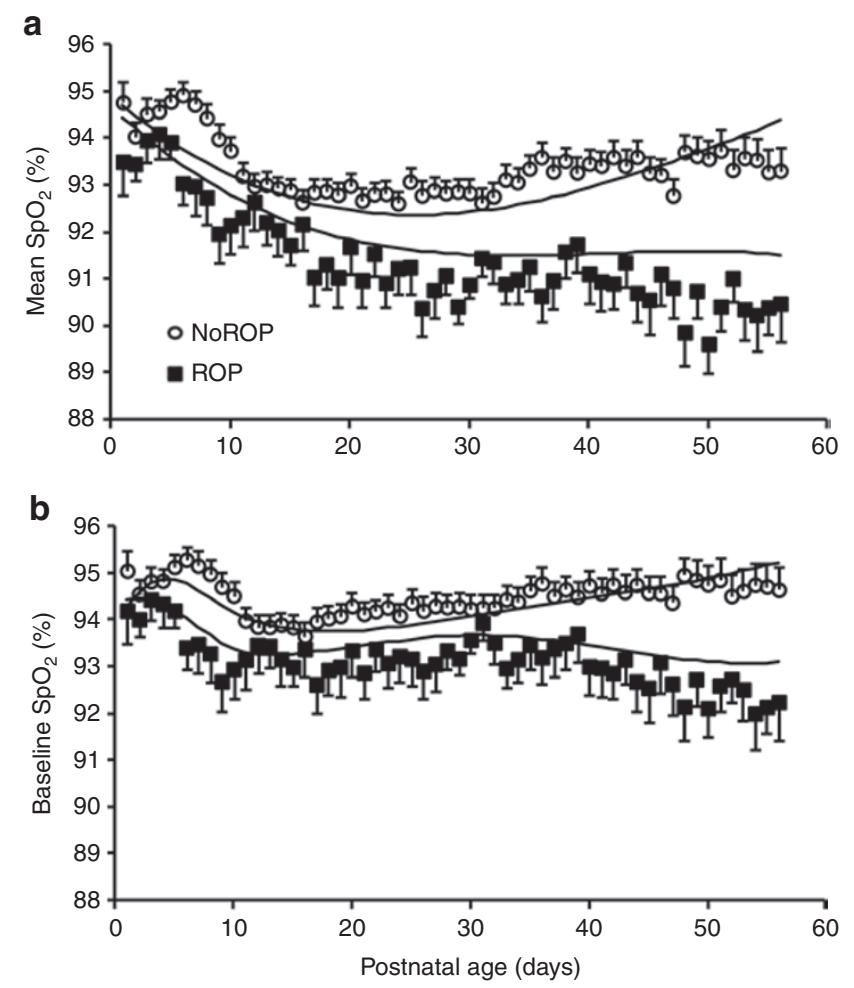

Figure 1. Mean and baseline $\mathrm{SpO}_{2}$ in infants with and without severe ROP. Both (a) mean $\mathrm{SpO}_{2}$ and (b) baseline $\mathrm{SpO}_{2}$, excluding $\mathrm{IH}$ events, were significantly lower in the severe ROP group $(P<0.05$ and $P<0.01$, respectively). Open circles, no or mild ROP; filled squares, severe ROP; solid line, covariate-adjusted models. Mean $\pm \mathrm{SEM}$. IH, intermittent hypoxemia; $\mathrm{ROP}$, retinopathy of prematurity; $\mathrm{SpO}_{2}$, oxygen saturation.

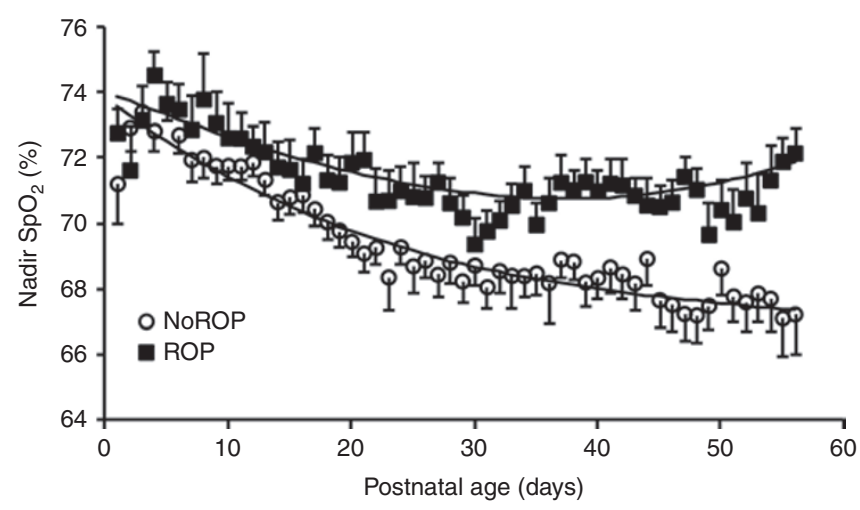

Figure 2. There was an increase in event severity with increasing age in both infant groups. Infants with severe ROP had less severe events, as defined by a higher nadir, after $14 \mathrm{~d}$ of life $(P<0.05)$. Open circles, no or mild ROP; filled squares, severe ROP; solid line, covariate-adjusted models. Mean \pm SEM. ROP, retinopathy of prematurity.

\section{Duration of IH Events}

The mean and SD of the duration of IH events decreased with increasing age in the no or mild ROP group. In contrast, both parameters remained constant in the severe ROP group, with a longer mean and higher $\mathrm{SD}$ as compared with the no or mild ROP group (both $P<0.005)$, with significantly longer mean durations $\geq 21 \mathrm{~d}$ of life $(P<0.005)$ and higher SDs of the duration of IH events $\geq 28 \mathrm{~d}$ of life $(P=0.004)$ (Figure 3$)$. There were no differences in the coefficient of variation of the IH event
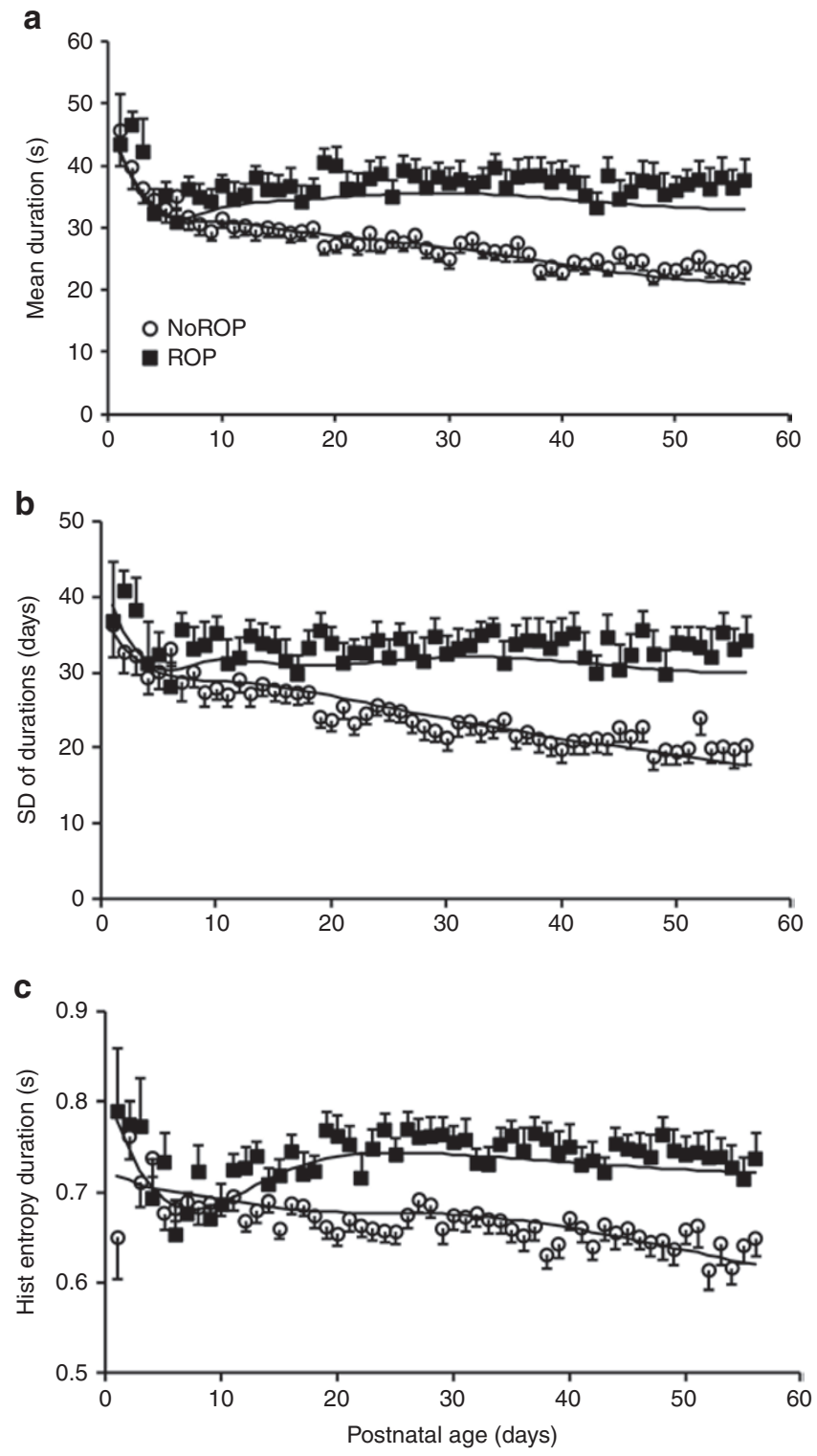

Figure 3. Mean and variability of $\mathrm{IH}$ duration in infants with and without severe ROP. The (a) mean, (b) SD, and (c) histogram (Hist) entropy of the duration of $\mathrm{IH}$ events decreased with increasing postnatal age in the no or mild ROP group. In contrast, these parameters remained relatively constant in the severe ROP group after the first week of life, with a longer mean $(P<0.05)$, higher SD $(P<0.05)$, and higher histogram entropy $(P<0.0001)$ as compared with the no or mild ROP group. Histogram entropy may be a more sensitive marker, with earlier differences identified between groups at $\geq 14 \mathrm{~d}$ of life $(P<0.05)$, followed by mean duration $\geq 21 \mathrm{~d}$ of life $(P<0.005)$ and SD of IH event durations at $\geq 28 \mathrm{~d}$ of life $(P=0.004)$. Open circles, no or mild ROP; filled squares, severe ROP; solid line, covariate-adjusted models. Mean \pm SEM. IH, intermittent hypoxemia; ROP, retinopathy of prematurity.

durations between infant groups (data not shown). Histogram entropy of the durations decreased with increasing postnatal age in the no or mild ROP group, indicating a trend toward reduced variability and complexity in the IH event time series. In contrast, histogram entropy was higher in the severe ROP group $(P<0.0001)$ and remained relatively constant, revealing a significantly less predictable pattern of event durations in that infant cohort at $\geq 14 \mathrm{~d}$ of life $(P<0.05)$ (Figure $3 \mathrm{c}$ ). 
a
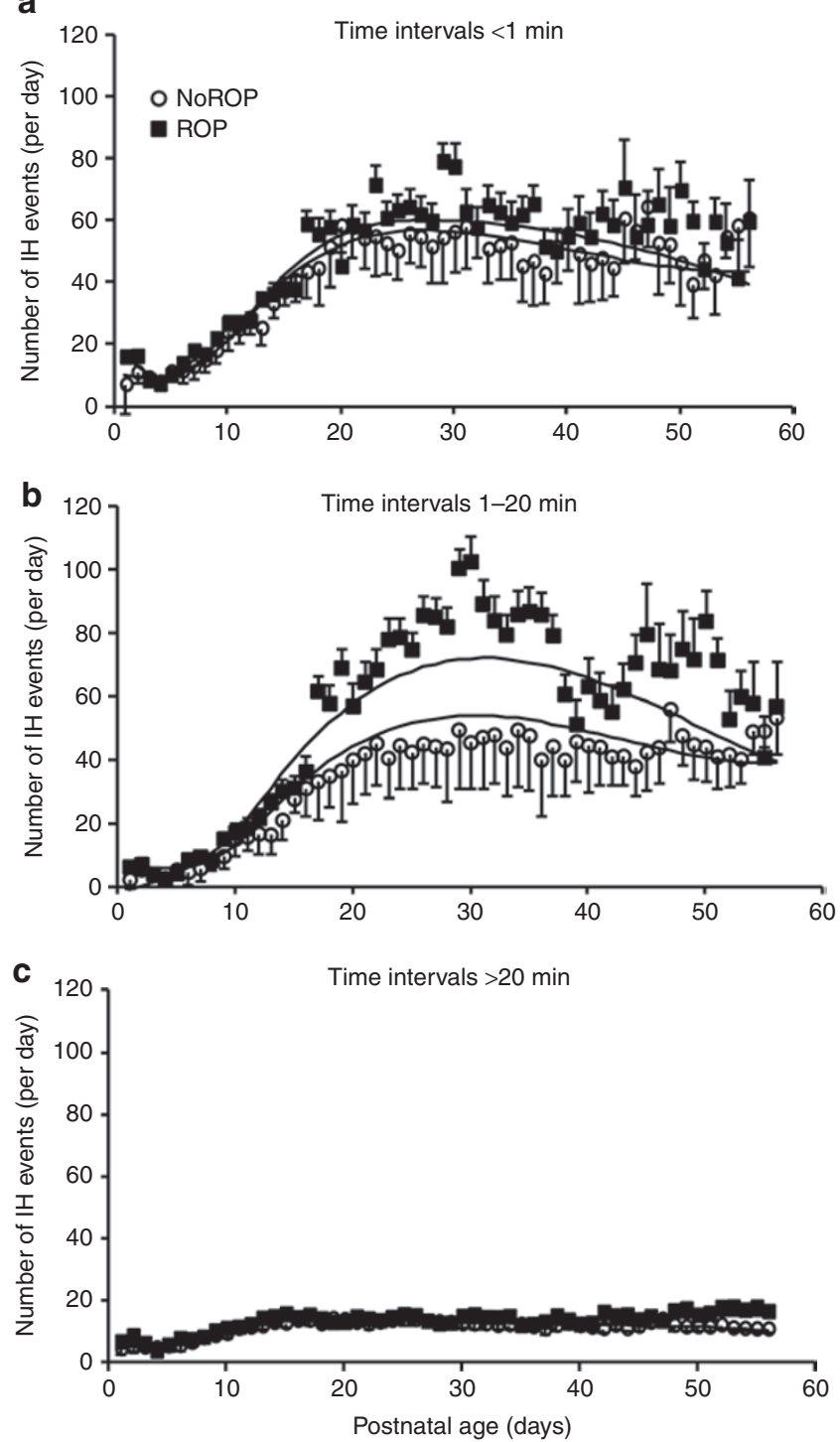

Figure 4. Stratification of events by a time interval between events of (a) $<1 \mathrm{~min}$, (b) 1-20 min, and (c) $>20 \mathrm{~min}$. (a) A substantial number of events occurred $<1$ min apart; however, there were no differences between infant groups. (b) A higher number of $\mathrm{IH}$ events in the severe ROP group was associated with a time interval of $1-20 \mathrm{~min}$ between IH events, with a significant difference at $28 \mathrm{~d}$ of life $(P<0.05)$ and a trend toward a higher frequency of events at 21 and $35 \mathrm{~d}$ (both $P=0.064$ ). (c) Relatively few IH events occurred with a time interval of $>20$ min between IH events, with no overall difference between infant groups. Open circles, no or mild ROP; filled squares, severe ROP; solid line, covariate-adjusted models. Mean \pm SEM. IH, intermittent hypoxemia; ROP, retinopathy of prematurity.

\section{Time Interval Between Events}

The mean and SD of the time interval between $\mathrm{IH}$ events declined over the first $3 \mathrm{wk}$ of life, with no differences between infant groups, and the coefficient of variation remained constant (data not shown). Histogram entropy of the time intervals was higher in the severe ROP group $(P<0.005)$, with significant differences between infant groups at $28 \mathrm{~d}$ life $(P=0.033)$.

Because of the wide variability in the time interval between $\mathrm{IH}$ events, the number of $\mathrm{IH}$ events was stratified into three time interval windows: those that occurred with a time interval

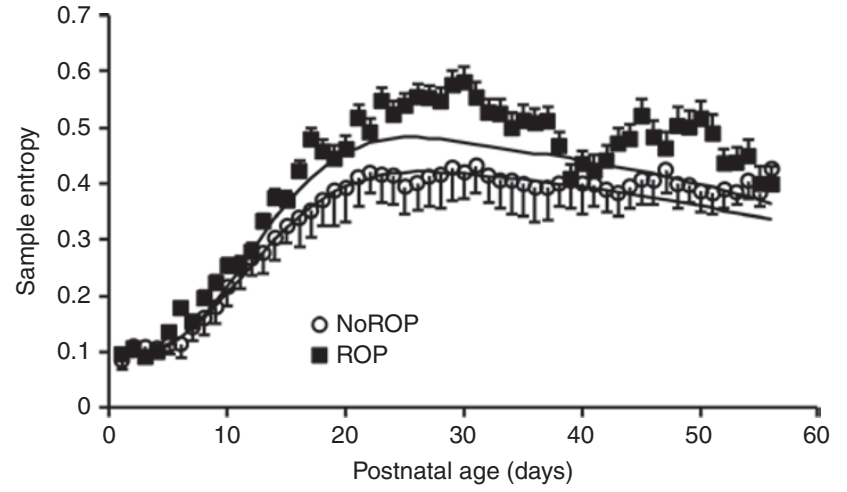

Figure 5. There was an increase in sample entropy with increasing postnatal age in both infant groups. Although there was a slightly higher sample entropy in the ROP group, there was no significant difference between groups. Open circles, no or mild ROP; filled squares, severe ROP; solid line, covariate-adjusted models. Mean \pm SEM. ROP, retinopathy of prematurity.

between events of $<1 \mathrm{~min}, 1-20 \mathrm{~min}$, and $>20 \mathrm{~min}$ (Figure 4 ). A substantial number of events occurred $<1$ min apart, however, there were no differences between infant groups at this time interval range. Relatively few $\mathrm{IH}$ events occurred with a time interval of $>20$ min between IH events, also with no overall difference between infant groups. Of note, the higher number of IH events in the severe ROP group was associated with a time interval of 1-20 min between IH events, significantly different at $28 \mathrm{~d}$ of life $(P<0.05)$ and with a trend toward a higher frequency of events at 21 and $35 \mathrm{~d}$ (both $P=0.064$ ).

\section{Oxygen Saturation Waveform Analysis}

To detect differences between groups that may have been missed by the arbitrary thresholds chosen to define an IH event, sample entropy (SamEn) and wavelet analyses were applied to the entire raw oxygen saturation $\left(\mathrm{SpO}_{2}\right)$ waveform. There was an increase in SamEn of the $\mathrm{SpO}_{2}$ waveform with increasing postnatal age in both infant groups, with no significant difference between groups (Figure 5). Wavelet analysis revealed differences in spectral power between the two infant groups that were frequency and age dependent (Figure 6). At higher frequencies (D1: $0.125-0.250 \mathrm{~Hz}, \mathrm{D} 2: 0.063-0.125 \mathrm{~Hz}$, and D3: $0.031-0.063 \mathrm{~Hz}$ ), the severe ROP infant group had significantly lower spectral power (all $P<0.05$ ) from 21 to $49 \mathrm{~d}$ of life at level D1 $(P<0.05)$ and at $\geq 14 \mathrm{~d}$ of life at levels D2 and D3 (both $P<0.05)$. In contrast, there was a shift toward a significantly higher spectral power in the severe ROP group at the two lowest frequency levels (D6: $0.004-0.008 \mathrm{~Hz}, 21-28 \mathrm{~d}$ of life $(P<0.05)$, D7: $0.002-0.004 \mathrm{~Hz}, 21-35 \mathrm{~d}$ of life $(P<0.05))$. Therefore, the ROP group had lower power in $\mathrm{SpO}_{2}$ waveform oscillations of 4-30 s in duration and higher power in $\mathrm{SpO}_{2}$ waveform oscillations of $2-8 \mathrm{~min}$ in duration.

\section{Postmenstrual Age}

In addition to the above analyses, which were based on postnatal age, we also compared IH events between ROP groups over postmenstrual age. There were IH events of longer duration at 
a

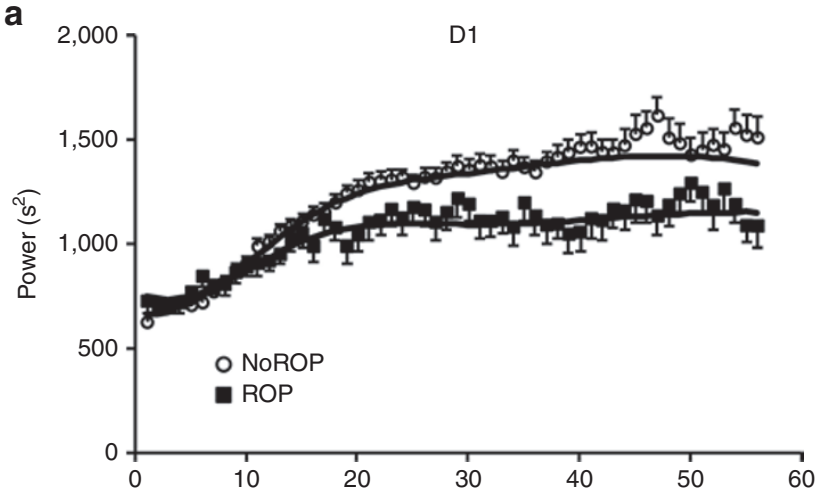

C

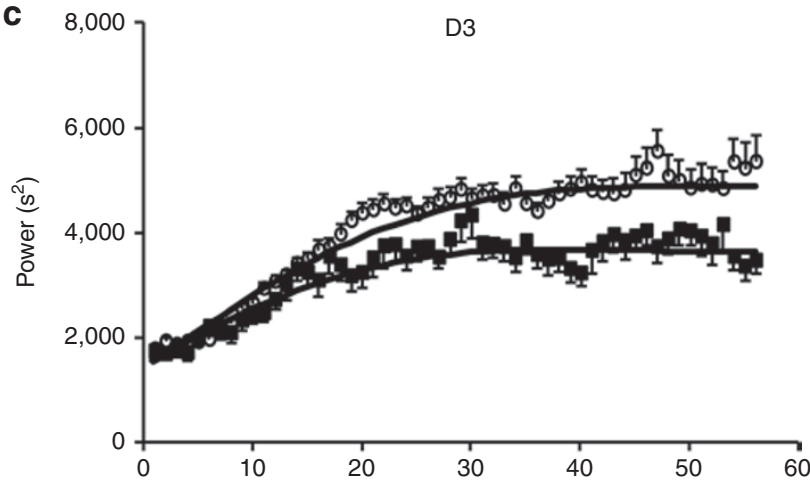

e

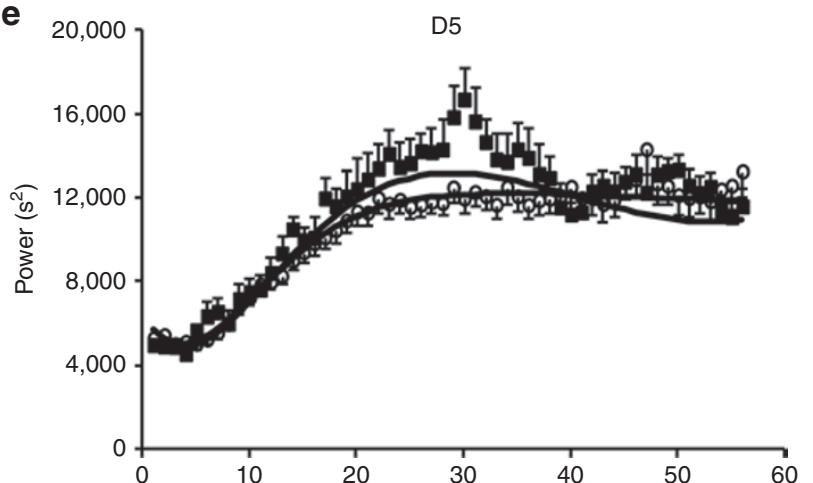

b

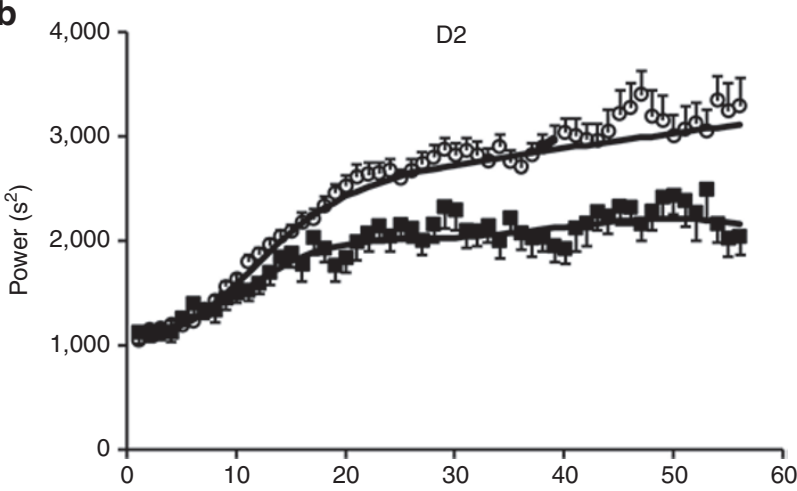

d

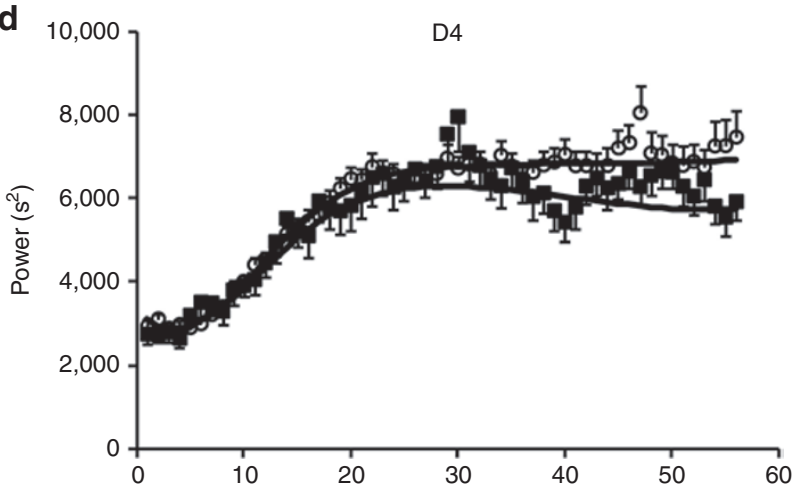

$\mathbf{f}$

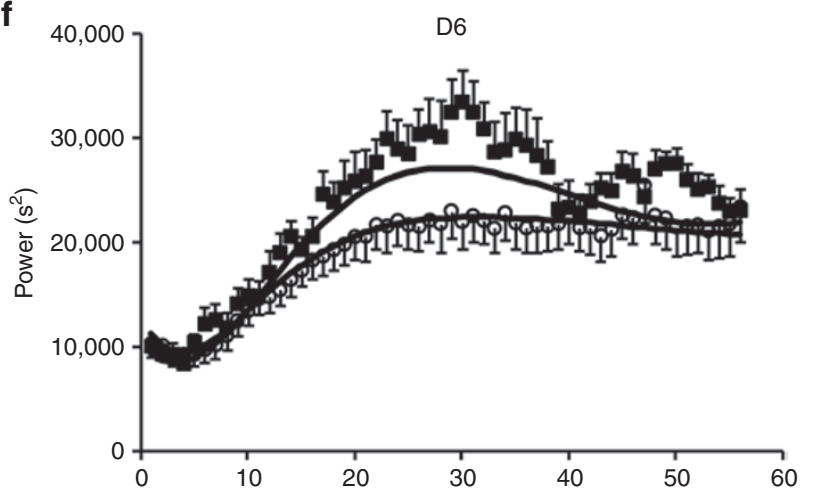

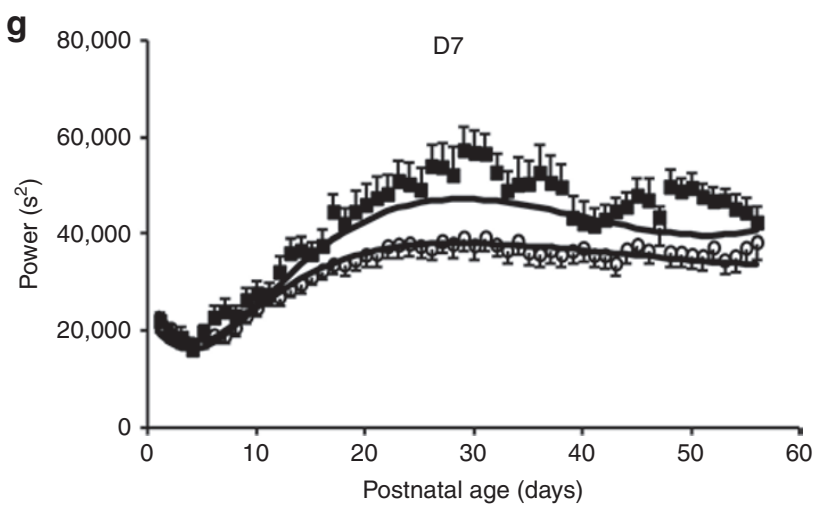

Figure 6. Wavelet analysis of the $\mathrm{SpO}_{2}$ waveform. At the higher frequencies levels, (a) D1: $0.125-0.250 \mathrm{~Hz}$, (b) D2: $0.063-0.125 \mathrm{~Hz}$, and (c) D3: $0.031-$ $0.063 \mathrm{~Hz}$, the severe ROP infant group had significantly lower spectral power from 21 to $49 \mathrm{~d}$ of life at level D1 $(P<0.05)$ and at $\geq 14 \mathrm{~d}$ of life at levels D2 and D3 (all $P<0.05$ ). There were no differences between groups at mid-frequencies (d) D4: 0.016-0.031 Hz and (e) D5: 0.008-0.016 Hz. At the two lowest frequency levels (f) D6: $0.004-0.008 \mathrm{~Hz}$ and $(\mathbf{g})$ D7: $0.002-0.004 \mathrm{~Hz}$, there was a shift toward a significantly higher spectral power in the severe ROP group from 21 to $28 \mathrm{~d}$ of life at level D6 $(P<0.05)$ and from 21 to $35 \mathrm{~d}$ of life at D7 $(P<0.05)$. Open circles, no or mild ROP; filled squares, severe ROP; solid line, covariate-adjusted models. Mean $\pm \mathrm{SEM}$. ROP, retinopathy of prematurity; $\mathrm{SpO}_{2^{\prime}}$ oxygen saturation. 


\section{Articles | Di Fiore et al.}

a postmenstrual age of $>28 \mathrm{wk}(P<0.05)$, and more IH events at 1-20 min intervals in the severe ROP group from 29 to $30 \mathrm{wk}$ $(P<0.05)$. There was lower power in the $\mathrm{D} 1$ frequency range and higher power in the D7 frequency range in the severe ROP group at $29-33 \mathrm{wk}(P<0.05)$ and $29-30 \mathrm{wk}(P<0.05)$ postmenstrual age, respectively.

\section{DISCUSSION}

Previous work by our group found more IH events in this cohort of preterm infants with severe ROP. This study gives novel new insights into the patterns of these events. Infants with severe ROP had longer, more variable, and less predictable IH event durations. Standard statistical summary measures (mean and $\mathrm{SD}$ ) were inadequate in their ability to distinguish group differences in IH event time intervals, but stratification of IH events by time interval ranges revealed a higher number of IH events in the severe ROP group associated with 1-20 min intervals between events. SamEn, an unbiased measure of predictability based on the entire $\mathrm{SpO}_{2}$ waveform data, was found to be slightly but not significantly increased in the severe ROP group. Finally, wavelet analysis revealed time-frequency-dependent differences in spectral power between infant groups. In summary, this study identifies potential tools that can be used to identify infants who may be at future risk for severe ROP based on dynamic temporal and time-frequency patterns of the oxygen saturation waveform.

Using standard statistical descriptors (mean and SD) and histogram entropy (8), we now show that infants with severe ROP have longer and less predictable IH event durations. This may be due to decreased oxygen reserve destabilizing respiration. A mathematical model incorporating pulmonary uptake dynamics found that low pre-apneic alveolar $\mathrm{PO}_{2}$ caused early onset of desaturation (9). The lower baseline oxygen saturation levels in the severe ROP group, which suggest a decreased oxygen reserve, may have initiated an early onset of desaturation and prolonged the duration of $\mathrm{IH}$ events in this infant cohort. The instability related to an immature respiratory system compounded by low baseline oxygen saturation may lead to more complex or variable duration patterns showing a higher histogram entropy value in the ROP group. Of note, even with longer, less predictable durations, the infants with ROP had less severe events. Nock et al. (10) showed an augmented ventilatory response to hypoxia in preterm infants with increased apnea. This might imply that IH events accompanying apnea may initiate a downstream protective cascade, although the differential effects of this response on the duration and severity of IH events are unknown.

Although ROP is associated predominantly with hyperoxia, infants with severe ROP were found to have a lower baseline $\mathrm{SpO}_{2}$ after $42 \mathrm{~d}$ of life. It may be that both infant groups were exposed to relative "hyperoxia" during early postnatal life; however, the subsequent lower baseline oxygen saturation in the ROP group exacerbated the rebound neovascularization, leading to increased ROP.

Although our ability to detect differences between infant groups in the overall time interval between events using the proposed mathematical models was limited, we were able to isolate a window of time between IH events of 1 and 20 min that was associated with severe ROP. Chronic intermittent hypoxia has been shown to increase oxidative stress in adults (11) and oxidative stress has been linked to ROP through mechanisms related to oxygenation of retinal tissue (12). In a rodent model, superoxide anion concentration, a marker of oxidative stress, has been shown to increase during the re-oxygenation phase following hypoxic exposure (13) with a delay in the response of a few minutes. This time span corresponds to the differences between groups seen in this study at a time interval between events of 1-20 min. Although there were a substantial number of events occurring $<1$ min apart, the incidence was comparable between groups, indicating that this time interval may not be long enough for an oxidative stress response, related to the initiation of the ROP cascade, to occur. This may also suggest that periodic breathing, associated with short intervals between IH events, may not play a role in the relationship between $\mathrm{IH}$ events and ROP.

In this study, quantification of possible patterns of oxygen saturation associated with ROP was limited by the arbitrarily chosen definition of an IH event. Therefore, SamEn and wavelet analyses were used to evaluate unbiased patterns of the entire $\mathrm{SpO}_{2}$ waveform. Both spectral and SamEn measurements have been shown to be useful tools in revealing respiratory patterns associated with morbidity (14) and maturation (15). SamEn has been used by our group and others in animal (16) and human models $(15,17)$ to assess respiratory and electroencephalogram arousal pattern variability. After minimizing the influence of autocorrelation on the SamEn calculations (18) and defining a constant tolerance $(S D=16)$ within and between infant groups, we have shown a trend toward a higher SamEn in the severe ROP group; however, this was not statistically significant. A larger sample size may be needed to detect these subtle pattern differences between groups.

The number of infants with severe ROP may be a limitation of this study given that significant differences were found between infant groups in demographics and clinical course. After controlling for gestational age, race, gender, and multiple births in the statistical model, we were still able to detect differences in patterns of $\mathrm{IH}$ events between infant groups. However, with respiratory support and oxygen changing on a day-by-day basis, we were unable to control for these variables in the analyses. Therefore, the results of this study reveal an association and not a causal relationship between $\mathrm{IH}$ events and severe ROP. To distinguish intermittent hypoxia from prolonged changes in baseline oxygenation, we prospectively defined our $\mathrm{IH}$ events by a duration range from $10 \mathrm{~s}$ to $3 \mathrm{~min}$; therefore, events $>3 \mathrm{~min}$ were not included in the analysis. Subsequent analysis of the data looking at $\mathrm{IH}$ events $>3 \mathrm{~min}$ shows that these events would account for a median of $5 \%$ and $3 \%$ of all events in the severe ROP and no or mild ROP groups, respectively. Therefore, we feel that inclusion of these events would have minimal impact on our findings. Finally, inclusion of infants with mild ROP in the control group may have affected our results. As there is interexaminer variability in this area, we chose what we thought to be the clearest distinction between groups. 


\section{Hypoxia and retinopathy of prematurity}

Wavelet analysis is the most promising tool, revealing distinguishing patterns between the infant groups that were frequency and age dependent. Infants with severe ROP have lower power in the $\mathrm{D} 1-\mathrm{D} 3$ frequency range associated with short $\mathrm{SpO}_{2}$ oscillations of 4-30 s, such as those occurring during periodic breathing or short apnea. In contrast, infants with severe ROP had increased power in the D6-D7 frequency range, associated with longer $\mathrm{SpO}_{2}$ oscillations of $2-8 \mathrm{~min}$. These frequency-dependent differences between groups were detected as early as $14 \mathrm{~d}$ of life. In contrast to adult rats, neonatal rats have been shown to have an enhanced carotid body response to acute hypoxia following chronic intermittent hypoxic exposure (19). This sensitized carotid body activity may lead to a change in gain of the central nervous system (20) with resultant overcompensation of ventilation. The ventilatory gain mismatch may drive $\mathrm{CO}_{2}$ down, leading to ventilatory depression with accompanying oscillations in oxygen saturation and may explain the increased power seen in the $\mathrm{SpO}_{2}$ oscillations of long duration. The wavelet analysis methodology uses the entire $\mathrm{SpO}_{2}$ waveform without the restriction of arbitrarily defined thresholds or durations of $\mathrm{IH}$ events, can identify early distinguishable patterns in the oxygen saturation waveform, and may prove useful in the neonatal intensive care unit setting to identify infants at risk for severe ROP.

Application of the mathematical models presented in this study requires higher sample rates and shorter averaging times than conventionally used in clinical practice and previous studies. Prior infant data have suggested a relationship between fluctuations in oxygenation and ROP, with interpretation of results limited by intermittent blood gas monitoring (21-23). This study included minimally processed (24) and highest-resolution data available from the Masimo pulse oximeter (Irvine, CA) with data continuously recorded over $8 \mathrm{wk}$ of life (7), allowing for identification of subtle $\mathrm{SpO}_{2}$ waveform patterns associated with ROP. Storage of high-resolution data is currently limited by the pulse oximeter data storage capacity. Further development of long-term data storage at the bedside, along with automated application of mathematical signal analysis algorithms, may lead to identification of these early morbidity markers in the clinical setting.

At-risk IH patterns were analyzed by both postnatal and postmenstrual age. Palmer et al. (25) found an association between both postnatal and postmenstrual age and onset of pre-threshold ROP, with less variability when data were analyzed by postmenstrual age. Although we also detected group differences in IH patterns by postmenstrual age, these differences corresponded to 28-35 d of life in our youngest infants, of $24 \mathrm{wk}$ gestation. In contrast, the postnatal age model comparisons revealed group differences as early as $14 \mathrm{~d}$ of life. Therefore, we believe postnatal age to be the superior model given that it may lead to earlier intervention in this infant cohort with an increased chance of preventing this morbidity.

In conclusion, infants with severe ROP have a higher incidence of IH events of longer, more variable and less predictable duration. These events are more likely to occur with a time interval between events of $1-20 \mathrm{~min}$. Infants who go on to have severe ROP have a slightly lower baseline $\mathrm{SpO}_{2}$, which may destabilize respiratory control and/or lead to more complex patterns of oxygen saturation that may be identified by more comprehensive data analysis models such as SamEn or wavelet analysis. The results of this study may elucidate early patterns of IH events that put infants at risk for future development of severe ROP.

\section{METHODS}

The study population was our prior cohort in which we characterized the incidence of $\mathrm{IH}$ events in preterm infants. It comprised 79 infants (gestational age, 24-27 6/7 wk) admitted to the Rainbow Babies \& Children's Hospital between 2005 and 2009. Infants with no congenital malformations were eligible for enrollment regardless of their need for ventilatory support or supplemental oxygen. Given that this study included previously acquired data, Rainbow Babies \& Children's Hospital Institutional Review Board approval and waiver of consent were obtained for the study.

Patients received normal clinical care and were kept in an oxygen saturation target range of $85-95 \%$. To minimize disparities in diagnosis of less severe forms of ROP, infants were stratified into two groups: those requiring laser therapy for severe ROP and those having no or mild ROP. The guidelines of the Early Treatment for Retinopathy of Prematurity Cooperative Trial were used to determine the need for laser treatment (26).

IH events were documented from continuous, high-resolution (2-s averaging and $0.5 \mathrm{~Hz}$ sampling rate) recordings of oxygen saturation (Radical, Masimo) over the first $8 \mathrm{wk}$ of life. To distinguish IH events from prolonged changes in baseline oxygenation, an IH event was defined as a fall in $\mathrm{SpO}_{2}$ to $\leq 80 \%$ for $\geq 10 \mathrm{~s}$ and $\leq 3 \mathrm{~min}$ in duration. Customized software was developed to identify the incidence, duration, severity (nadir), and the time interval between IH events. A suite of linear and nonlinear analysis software algorithms was developed to quantify patterns of IH events including mean, SD, and coefficient of variation to quantify changes in frequency and variability of $\mathrm{IH}$ events, and algorithms derived from time-frequency signal analysis (wavelets), information theory (histogram entropy), and statistical signal processing (SamEn), were used to quantify different aspects of $\mathrm{IH}$ pattern variability. During the monitoring periods, there were intermittent times when signal loss occurred due to infants not being monitored, corrupt files, and noise or motion artifacts. Periods with $0 \% \mathrm{SpO}_{2}$ or $0 \mathrm{bpm}$ for heart rate were considered artifacts and removed from the data stream with the remaining data analyzed in $24 \mathrm{~h}$ (mean, SD, coefficient of variation, and histogram entropy) or 1-h (SamEn and wavelet analysis) sliding windows. Large areas of missing data, i.e., $24 \mathrm{~h}$, were treated as missing cells in the statistical analysis.

Histogram entropy was used to further understand how the patterning of the duration and time interval between hypoxic events influences the development of ROP. This measure can be used to quantify the degree of randomness in the data. For example, a signal with higher histogram entropy has a wider distribution of values, meaning the data are more variable and, thus, less predictable. The uniform distribution would have maximal histogram entropy. Accurate computation of histogram entropy requires a reliable distribution of the dataset; therefore, days with $<10$ events were excluded from this particular analysis.

Although histogram entropy is used for analyses in which variables can be sorted, such as IH event durations or time intervals between events, SamEn is used for continuous waveform data. Similar to histogram entropy, SamEn quantifies predictability in time series data based on the likelihood that a given window of $\mathrm{SpO}_{2}$ data will, over time, remain close to the last window of $\mathrm{SpO}_{2}$ data-e.g., given $1 \mathrm{~h}$ of $\mathrm{SpO}_{2}$ data, will the next $1 \mathrm{~h}$ window of data be similar in extent and variability? Examining this measure across groups offers a relative comparison of the "complexity" of the time series with higher values of SamEn associated with less predictability or higher system complexity. SamEn was computed using the formula:

$$
\operatorname{SamEn}(m, r, n)=-\ln \frac{A}{B}=-\ln \frac{\sum_{i=1}^{n-m \tau} A_{i}}{\sum_{i=1}^{n-m \tau} B_{i}}
$$


where $m=$ pattern length, $r=$ the tolerance parameter, $n=$ number of samples, $\tau=$ time delay, $A_{i}=$ the number of points in the time series within tolerance $r$ for the $m+1$-dimensional pattern, and $B_{i}=$ the number of points in the time series within tolerance $r$ for the $m$-dimensional pattern. SamEn was calculated using $m=2, r=0.2 \times$ SD, and $\tau=20$ samples. A nonunity time delay ( $\tau$ was chosen to reduce the contribution of the linear statistical autocorrelation properties of the signal (18). The value of $\tau=20$ samples was chosen based on pilot data in 10 infants identifying a time delay where the linear (auto)correlation was minimized. Because SamEn detects $\mathrm{SpO}_{2}$ patterns falling within a given tolerance $(r=0.2 \times \mathrm{SD})$, the same tolerance criteria were used for comparison within and between infants. A constant SD of 16 was chosen based on the maximum $\mathrm{SD}$ of $\mathrm{SpO}_{2}$ values for all days in all infants.

When viewing an overall $\mathrm{SpO}_{2}$ waveform over time, it can be seen that the waveform consists of oscillations throughout both "normal" and "hypoxic" ranges of $\mathrm{SpO}_{2}$. These oscillations can be decomposed into separate frequency components, using time-frequency (wavelet) analysis, allowing for quantifiable comparisons of the power of individual frequency ranges between the two infant groups. A seven-level 1-D stationary wavelet decomposition was performed using a Daubechies (fourth in the series) orthogonal wavelet decomposition filter over the entire waveform time series. The total power in each frequency range (D1: $0.125-0.250 \mathrm{~Hz}, \mathrm{D} 2: 0.063-0.125 \mathrm{~Hz}, \mathrm{D} 3: 0.031-0.063 \mathrm{~Hz}, \mathrm{D} 4$ : 0.016-0.031 Hz, D5: 0.008-0.016 Hz, D6: 0.004-0.008 Hz, D7: 0.002$0.004 \mathrm{~Hz}$, and A7: 0-0.002 Hz) was calculated for each 1-h window. Power in the D1-D3 frequency range corresponds to $\mathrm{SpO}$, waveform oscillations of 4-30 s in duration, such as those associated with periodic breathing or short apnea, whereas power in the D6-D7 frequency range corresponds to longer $\mathrm{SpO}_{2}$ waveform oscillations of 2-8 min. Data were analyzed, using both wavelet and SamEn algorithms, in 1-h sliding windows with no overlap, and windows with missing data were excluded from the analyses.

Statistical analysis of all measures was performed on daily averages computed for each subject from the hourly data. A logarithmic or square root transformation was applied when the original data were skewed. A $t$-test was used for demographic comparisons between infant groups. Data are presented as mean \pm SEM. Longitudinal profiles of severe ROP and no or mild ROP groups were estimated and compared using linear mixed models, modeling the nonlinear relationship of each outcome with age using cubic splines with knots at 10 , 14 , and $30 \mathrm{~d}$ (27). Simpler quadratic or cubic models were used for five variables (entropy of the time intervals, mean $\mathrm{SpO}_{2}$, nadir, $\mathrm{IH}$ events with time interval of 1-20 min, wavelet D1, and wavelet D2). Models included terms for gestational age, race, gender, multiple births, and their interactions with terms involving day. A likelihood ratio test was used to compare overall mean curves of severe ROP vs. no or mild $\mathrm{ROP}$ with post comparisons at days $1,7,14, \ldots, 56$. Analyses were conducted using SAS version 9.2 (SAS Institute, Cary, NC).

\section{STATEMENT OF FINANCIAL SUPPORT}

This study was supported by Eunice Kennedy Shriver National Institute of Child Health and Human Development grant 1RO3HD064830-01.

\section{REFERENCES}

1. SUPPORT Study Group of the Eunice Kennedy Shriver NICHD Neonatal Research Network. Target ranges of oxygen saturation in extremely preterm infants. N Eng J Med 2010;362:1959-69.

2. Chow LC, Wright KW, Sola A; CSMC Oxygen Administration Study Group. Can changes in clinical practice decrease the incidence of severe retinopathy of prematurity in very low birth weight infants? Pediatrics 2003;111:339-45.

3. Sears JE, Pietz J, Sonnie C, Dolcini D, Hoppe G. A change in oxygen supplementation can decrease the incidence of retinopathy of prematurity. Ophthalmology 2009;116:513-8.

4. Coleman RJ, Beharry KD, Brock RS, Abad-Santos P, Abad-Santos M, Modanlou HD. Effects of brief, clustered versus dispersed hypoxic episodes on systemic and ocular growth factors in a rat model of oxygeninduced retinopathy. Pediatr Res 2008;64:50-5.
5. Penn JS, Henry MM, Tolman BL. Exposure to alternating hypoxia and hyperoxia causes severe proliferative retinopathy in the newborn rat. Pediatr Res 1994;36:724-31.

6. Werdich XQ, McCollum GW, Rajaratnam VS, Penn JS. Variable oxygen and retinal VEGF levels: correlation with incidence and severity of pathology in a rat model of oxygen-induced retinopathy. Exp Eye Res 2004;79:623-30.

7. Di Fiore JM, Bloom JN, Orge F, et al. A higher incidence of intermittent hypoxemic episodes is associated with severe retinopathy of prematurity. J Pediatr 2010;157:69-73.

8. Shannon CE. A mathematical theory of communication. Bell Syst Tech J 1948;27:379-423, 623-56.

9. Sands SA, Edwards BA, Kelly VJ, Davidson MR, Wilkinson MH, Berger PJ. A model analysis of arterial oxygen desaturation during apnea in preterm infants. PLoS Comput Biol 2009;5:e1000588.

10. Nock ML, Di Fiore JM, Arko MK, Martin RJ. Relationship of the ventilatory response to hypoxia with neonatal apnea in preterm infants. J Pediatr 2004;144:291-5.

11. Pialoux V, Hanly PJ, Foster GE, et al. Effects of exposure to intermittent hypoxia on oxidative stress and acute hypoxic ventilatory response in humans. Am J Respir Crit Care Med 2009;180:1002-9.

12. Hartnett ME. The effects of oxygen stresses on the development of features of severe retinopathy of prematurity: knowledge from the 50/10 OIR model. Doc Ophthalmol 2010;120:25-39.

13. Fabian RH, Perez-Polo JR, Kent TA. Extracellular superoxide concentration increases following cerebral hypoxia but does not affect cerebral blood flow. Int J Dev Neurosci 2004;22:225-30.

14. Marchenko V, Rogers RF. Time-frequency coherence analysis of phrenic and hypoglossal activity in the decerebrate rat during eupnea, hyperpnea, and gasping. Am J Physiol Regul Integr Comp Physiol 2006;291:R1430-42.

15. Engoren M, Courtney SE, Habib RH. Effect of weight and age on respiratory complexity in premature neonates. J Appl Physiol 2009;106:766-73.

16. Dragomir A, Akay Y, Curran AK, Akay M. Investigating the complexity of respiratory patterns during the laryngeal chemoreflex. J Neuroeng Rehabil 2008;5:17.

17. Jamasebi R, Redline S, Patel SR, Loparo KA. Entropy-based measures of EEG arousals as biomarkers for sleep dynamics: applications to hypertension. Sleep 2008;31:935-43.

18. Kaffashi F, Fogylano R, Wilson CG, Loparo KA. The effect of time on approximate and sample entropy calculations. Physica D 2008;237:3069-74.

19. Pawar A, Peng YJ, Jacono FJ, Prabhakar NR. Comparative analysis of neonatal and adult rat carotid body responses to chronic intermittent hypoxia. J Appl Physiol 2008;104:1287-94.

20. Smith CA, Forster HV, Blain GM, Dempsey JA. An interdependent model of central/peripheral chemoreception: evidence and implications for ventilatory control. Respir Physiol Neurobiol 2010;173:288-97.

21. York JR, Landers S, Kirby RS, Arbogast PG, Penn JS. Arterial oxygen fluctuation and retinopathy of prematurity in very-low-birth-weight infants. J Perinatol 2004;24:82-7.

22. Saito Y, Omoto T, Cho Y, Hatsukawa Y, Fujimura M, Takeuchi T. The progression of retinopathy of prematurity and fluctuation in blood gas tension. Graefes Arch Clin Exp Ophthalmol 1993;231:151-6.

23. Hauspurg AK, Allred AN, Vanderveen DK, et al. Blood gases and retinopathy of prematurity: the ELGAN study. Neonatol 2011;99:104-11.

24. Farré R, Montserrat JM, Ballester E, Hernández L, Rotger M, Navajas D. Importance of the pulse oximeter averaging time when measuring oxygen desaturation in sleep apnea. Sleep 1998;21:386-90.

25. Palmer EA, Flynn JT, Hardy RJ, et al. Incidence and early course of retinopathy of prematurity. The Cryotherapy for Retinopathy of Prematurity Cooperative Group. Ophthalmology 1991;98:1628-40.

26. Early Treatment For Retinopathy Of Prematurity Cooperative Group. Revised indications for the treatment of retinopathy of prematurity: results of the early treatment for retinopathy of prematurity randomized trial. Arch Ophthalmol 2003;121:1684-94.

27. Harrell FE. Regression Modeling Strategies with Applications to Linear Models, Logistic Regression, and Survival Analysis. New York: Springer, 2001. 